\title{
Performance Analysis of Wireless Mobile Adhoc Network with Different Types of Antennas
}

\author{
Monika Bansal1), Laxmi Shricastava2)
}

\begin{abstract}
The mobile ad network has become a new area in the communication networks and is being congested due to viability and utility of the ad hoc networks in every area. The wide use has increased the chances of interference and congestion. Use of directional antennas can largely reduce the radio interference, hence improving the utilization of wireless medium and consequently the network throughput. In this paper the Performance of ad-hoc networks has been analysed by using various types of antennas. The result has been analysed for different types and can help in deciding the type of antenna to be used depending on radio noise conditions.
\end{abstract}

Keywords: antennas, wireless, mobile, adhoc, performance analysis, smart antenna.

\section{Introduction}

Ad hoc wireless networks [1][2] are considered to be an infrastructure-less networks where each node is capable of self routing and is considered to be a mobile router, and is equipped with a wireless transceiver. The nodes in this network are autonomous, which share a single wireless channel for the purpose of communicating with each other. The first stage of ad hoc networks were generally using omni-directional antennas, which used to transmit or receive the radio energy to and from all directions. In the second stage the network started using smart antenna technology, which may have significant potentials to make the transmission in specific direction at a time[3]. The use of directional antenna improves spatial reuse of the wireless

Received(November 29, 2016), Review Result(1st: December 14, 2016, 2nd: January 18, 2017), Accepted(March 10, 2017)

${ }^{1}$ Vetri Vikas Educational Institutions, Mallur (Via), Rasipuram Taluk, Annamalaipatti, Tamil Nadu, India email: bansalmonika094@gmail.com

${ }^{2}$ (Corresponding Author) Vetri Vikas Educational Institutions, Mallur (Via), Rasipuram Taluk, Annamalaipatti, Tamil Nadu, India email: 1selex@yahoo.com 
channel, and allows network nodes to communicate at a time with reduced interference and more pairs in the same region. The other advantage of using directional transmission is to have communication with more range using less transmission power as transmission concentrates signal power to the receiver[4-6]. In process, it can establish links between nodes having large distance then using omnidirectional, and it also prevents network partitions and the number of routing hops can be lesser than those of omni-directional communication.

\section{Antenna Types}

\subsection{Directional Antennas}

The directional antennas are designed radiate in particular direction and the radiating area is decided by the design having certain degree of beam width. The particular direction radiation requires less energy but restricts the coverage area[7-9]. However, it helps in spatial reuse and energy conservation in the network area. The antennas used in the network design are being discussed in the coming section[10-12]. The Antennas and their classification are dependent on the parameters of performance and are classified on various parameters, which are discussed in the next section.

The classification of antennas is shown in Figure 1. Among various antenna types, Omnidirectional antennas are commonly used in wireless networks.

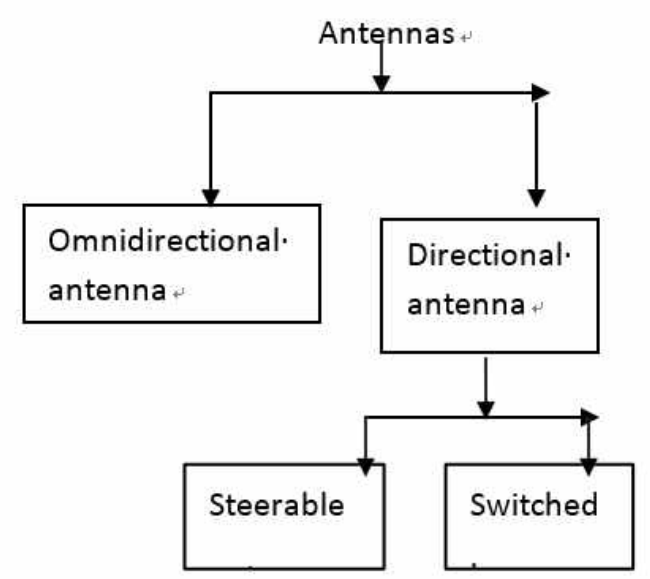

[Fig. 1] Types of Antenna Directional Antenna

\subsection{Omnidirectional Antennas}


The basic antenna used for communication since beginning of wireless communication is omni-directional antenna, which is also named as isotropic, and is considered to be a point in space, which radiates energy in all directions equally in to stablish wireless communication between transmitter and receiver. This type of antenna is referred as ideal antenna and is taken as a reference model of all the practical antennas designed[13][14]. A half-wave dipole is a practical omni-directional antenna, which has a circular radiation pattern in the azimuth plane or in the elevation plane. As an omni-directional antenna radiates radio signals in all directions, only a small percentage of them can reach the desired nodes and most of them are scattered into space. The scattered radio energy causes interference with other nodes residing in the coverage of the antenna. Apart from this the omni-directional antennas have very low effi ciency in the frequency reuse as it needs more number of channels are required to avoid interference.

A directional antenna is an antenna or may be an antenna array, which can radiate or receive radio signals more effectively in specific directions with fixed angle than in others. There are two kinds of directional antennas: Traditional directed antennas include Helix, Yagi-Uda, aperture horn, reflector (e.g., parabolic grid antenna), and patch antennas. The beam formed by the antennas is either fixed or adjusted to point to a certain direction by mechanical rotation. The antennas are designed on the basis of coverage area and having specific beam angle of radiation.

\subsection{Smart Antenna}

A smart antenna is an antenna array, which consists of a set of antenna elements in a linear, planar, or circular array having capability of digital signal processing to transmit and receive the energy adaptively. Compared with traditional directed antennas, smart antennas usually possess superior capabilities, such as directional beam forming, diversity processing, and adaptive spatial reusing. A smart antenna is composed of a number of radiating elements and a combining/dividing network and a control unit. The control unit that is normally implemented by using a digital signal processor (DSP) is the brain of a smart antenna. Many terms have been used to refer to smart antennas, which include intelligent antennas, phased array, spatial processing, digital beam forming, and adaptive antenna systems. The smart antennas can be categorized as the following types.

\subsection{Switched Beam}


In this type of smart antenna the beam patterns are predetermined by shifting every antenna element's signal phase depending its coverage area and defining the radiation angle. Weights for antenna elements, which are used to produce the desired beam pattern, can be locally saved in memory and instantaneously switched.

\subsection{Steerable Single Beam Antenna}

In this system, arbitrary beam patterns are formed on the fly. Besides, the radiation pattern can be adjusted toward the users whereas directions toward interference sources are set to be null (or zero), which is called nulling capability. These techniques can maximize the signal to interference and noise ratio (SINR)[15].

\subsection{Radiation patterns for directional antennas}

The radiation pattern of an antenna is the $2 \mathrm{D}$ or $3 \mathrm{D}$ spatial distribution of antenna gain as a function of the observer's position along a path or surface of constant radius. As the radiation pattern of a directional antenna is too complicated to be used in analysis, there are two simpli fied models for radiation patterns of directional antennas:

Flat-top radiation pattern: This model assumes that the antenna gain is constant within the main beam with beamwidth and both sidelobes and backlobes are ignored (i.e., the gain outside the beamwidth is regarded as zero)

$$
\mathrm{G}=2 \pi / \theta
$$

Cone+Sphere radiation pattern: The antenna pattern for the antenna gain consists of a main lobe with the beamwidth and a side lobe with a beamwidth. The main lobe is modeled as a cone of uniform gain, and the side lobes are aggregated to a single bulb at the base of the cone.

\section{Performance Metrices}

Some of the important performance metrics can be evaluated. 
1. Throughput: The average rate of data successfully received at the destination from the source is throughput. It is usually measured in bits per sec (bit/s or bps), and sometimes in data packets per second.

2. Average end to end delay: The difference in the calculation while transmitting, packets send time and received time is average end to end delay. This delay is generally due to route discovery, re-transmission delay and queuing propagation.

3. Jitter: Jitter is defined as a variation in the delay of received packets. The sending side transmits packets in a continuous stream and spaces them evenly apart. Because ofnetwork congestion, improper queuing,or configuration errors, the delay between packets can vary instead of remaining constant.

4. PDR: Packet delivery ratio is defined as the ratio of data packets received by the destinations to those generated by the sources.

\section{Simulation Setup and Environment}

The simulation model was developed in the scalable and portable simulator qualnet 6.1 with the features supporting various directional antennas. For the simulation setup we took 50 mobile nodes randomly placed in a terrain size of 1500mx1500m (Fig.1). The mobility model and the energy model used are random way point and mica $\mathrm{z}$ respectively. The battery model and propagation model used is linear model and rayiegh model respectively. For the traffic generation, the traffic source used is CBR (constant bit rate) in which 512 bytes of data at 2 Mbps rate is sent over the network. The design scenario of 50 nodes randomly placed in the defined terrain is shown below in figure 2.

There are different default values and user defined values are taken in carrying out simulations. The simulation parameters and the antenna parameters are summarized below in Table 1 and Table 2 respectively.

\section{Simulation results}

In this paper three scenarios have been simulated using qualnet simulation software. In the first scenario, all 50 nodes are equipped with omni directional antennas and reactive routing 
Performance Analysis of Wireless Mobile Adhoc Network with Different Types of Antennas

protocols (AODV) is used. In the second scenario, all the nodes are equipped with steerable antennas. In the third scenario, all the 50 nodes are equipped with switched beam antennas and the AODV routing protocol is used.

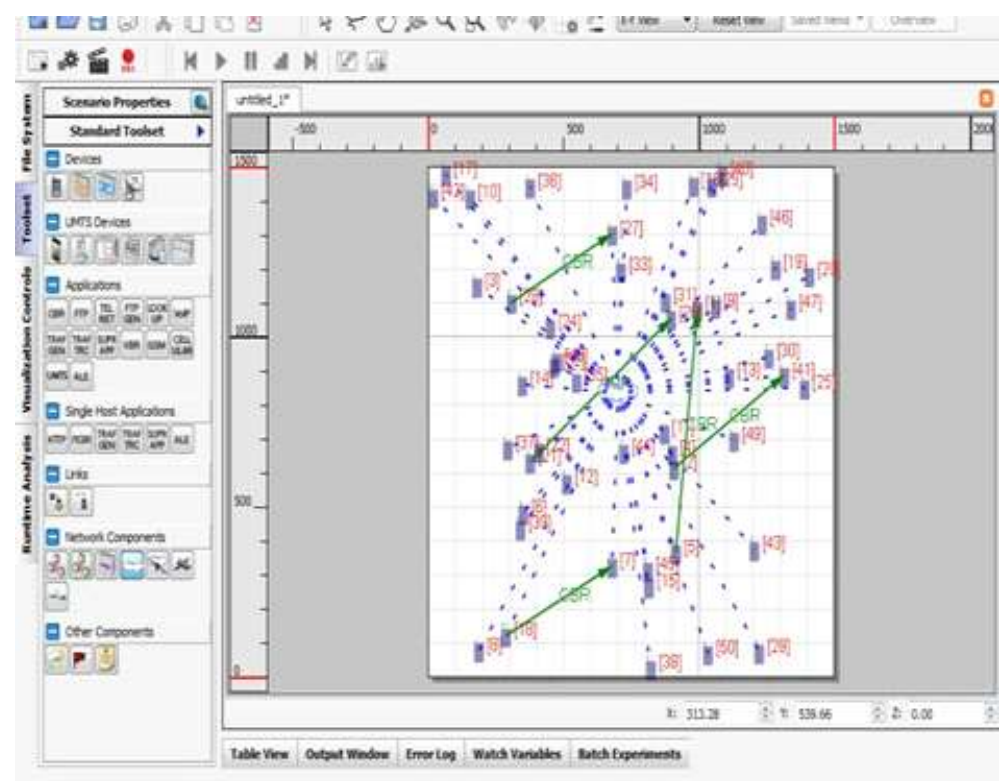

[Fig. 2] Design scenario of 50 nodes placed randomly

[Table 1] Simulation parameters

\begin{tabular}{|l|l|}
\hline Parameters & Values \\
\hline Simulator & Qualnet6.1 \\
\hline Terrain Size & $1500 \mathrm{mx} 1500 \mathrm{~m}$ \\
\hline No. of nodes & 50 \\
\hline Network protocol & IPV4 \\
\hline Data size & 512 Bytes \\
\hline Data rates & $2 \mathrm{mbps}$ \\
\hline Mobility model & Random way point \\
\hline Propagation model & Rayliegh \\
\hline Channel frequency & $2.4 \mathrm{Ghz}$ \\
\hline Traffic source & CBR \\
\hline Simulaton time & 250 sec \\
\hline Routig protocol & Bellman Ford \\
\hline
\end{tabular}


[Table 2] Antenna parameters

\begin{tabular}{|l|l|l|l|}
\hline Parameters & Values \\
\hline Antenna Model & Omni directional & Steerable & Switched beam \\
\hline Antenna Gain & $1.0 \mathrm{db}$ & 15.268 & 15.268 \\
\hline Azimuthal angles & $0 \mathrm{degree}$ & $60 \mathrm{deg}$ & $60 \mathrm{deg}$ \\
\hline Antenna height & $1.5 \mathrm{~m}$ & \\
\hline Antenna efficiency & 0.8 & \\
\hline Antenna mismatch loss & $0.3 \mathrm{db}$ & \\
\hline Antenna Connection loss & $0.2 \mathrm{db}$ & \\
\hline Antenna Cable loss & $0.01 \mathrm{db}$ & \\
\hline Noise factor & 10.0 &
\end{tabular}

The main purpose of the above simulation is to compare various QoS parameters like average Throughput, average end to end delay, average jitter, packet delivery ratio in wireless MANET for omnidirectional antennas and both the above mentioned directional antennas. The overall simulation results are shown in table 3 .

[Table 3] Simulation results

\begin{tabular}{|l|l|l|l|}
\hline \multirow{2}{*}{ QoS Parameter s } & Antenna model used \\
\cline { 2 - 4 } & $\begin{array}{l}\text { Omni } \\
\text { directional }\end{array}$ & $\begin{array}{l}\text { Steerable } \\
\text { antenna }\end{array}$ & $\begin{array}{l}\text { Switched } \\
\text { Antenna }\end{array}$ \\
\hline Average Throughput (bps) & 4005.67 & 4274.83 & 4276.93 \\
\hline Average End to End Delay(sec) & 0.035214 & 0.025817 & 0.027863 \\
\hline Average Jitter(sec) & 0.007981 & 0.00561 & 0.006913 \\
\hline Packet Delivery Ratio(\%) & 85 & 95 & 96 \\
\hline
\end{tabular}

According to the above simulation results, comparisons of the various parameters were carried out between omni directional antennas and directional antennas like steerable and switched beam antennas. The comparison is done by the help of bar graphs shown below.

Fig.3 shows the comparison o the average value of throughput for MANET using the said Omni and directional antennas. From the results plotted on the graph we find that the highest 
average value of throughput is achieved in case of steerable and switched beam directional antennas whereas in case of Omni directional antennas the value of throughput is comparatively very less.

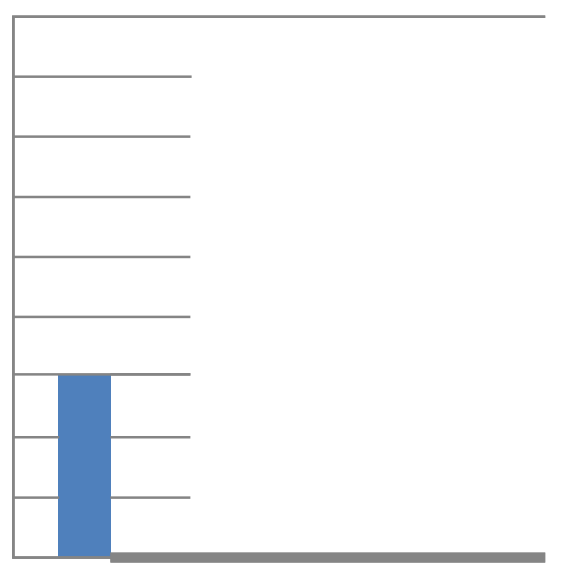

[Fig. 3] Comparison of Average Throughput between Omni directional, steerable and switched beam antennas

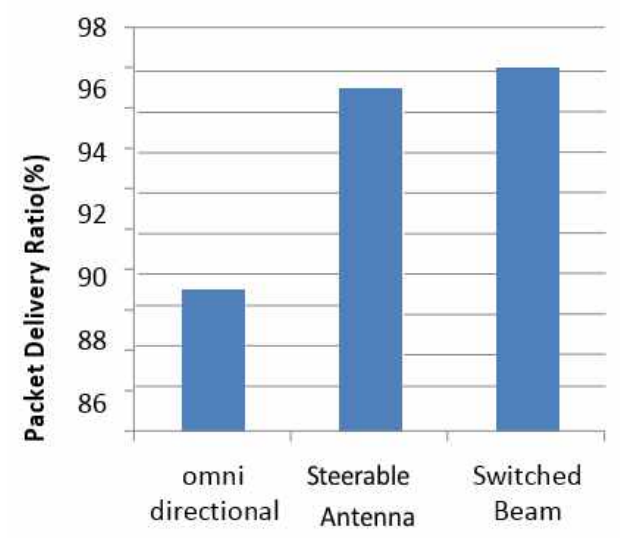

[Fig.4] Comparison f Average end to end delay between Omni directional, steerable and switched beam antennas

In Fig. 4, we can see the comparison of the value of average end to end delay among Omni directional, steerable and switched Beam antennas. From the results, we find that the smallest value of average end to end delay is achieved only in case of directional antennas (steerable) as compared with the Omni directional antennas.

Fig 5. contains the comparison of the values of average jitter between omni directional and two directional antennas. The lowest value of average jitter was achieved in case of directional antennas(steerable) as compared with the omni directional antenna. 


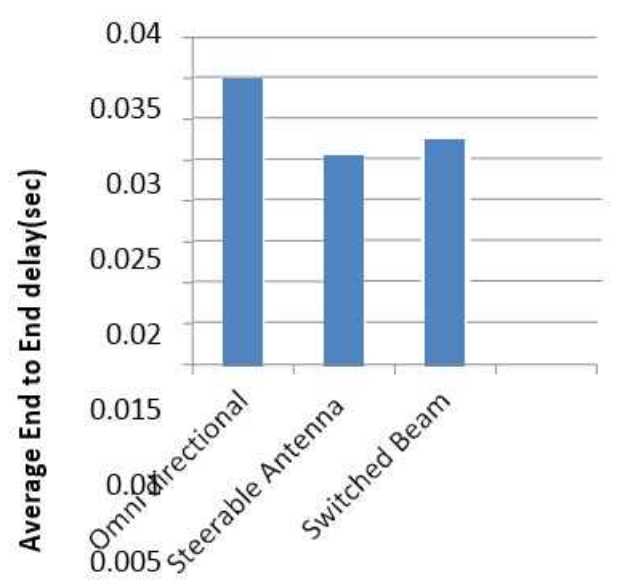

[Fig 5] Comparison of Average Jitter between omni directional, Steerable and Switched beam antennas

We noticed that for steerable and switched beam antennas the packet delivery ratio is approx $100 \%$ whereas the Omnidirectional antenna produced $91.67 \%$. It means there is a less chance of packet drop.

\section{Conclusion}

In this paper the comparative study has been presented on the basis of three simulation carried out using Omni directional, steerable and switched beam antennas and results have been compared on the basis of various parameters deciding the quality of the network. The QoS parameters like Average throughput, Average End to End delay, Average Jitter, and Packet Delivery ratio were chosen to check the performance and efficiency of the network in different scenarios. In the first simulation scenario Omni directional antenna was used for all nodes in the network whereas, for the second and third simulation scenario steerable and switched beam directional antennas were used for all the nodes in the network respectively. From the above results, we can see that in case of Average throughput, both the directional antenna produce high throughput than the omni directional antenna. And in case of Average End to End delay and Average jitter, the delay and Jitter is high in case of omni directional antenna as compared with steerable antenna and switched beam antennas. Directional antennas like steerable and switched beam are more efficient in sending packets correctly at the destination because from the above results the Packet Delivery Ratio is $95 \%$ and $96 \%$ in case of directional 
antennas whereas only $85 \%$ in case of omni directional antenna.

So from the above results, we can conclude that by using directional antennas the Quality of Service (QoS) parameters can be improved and enhanced but there is always some limitation and challenges in the MAC layer and Physical layer which affect the performance of the directional antennas.

\section{References}

[1] R. Ramanathan, On the Performance of Ad Hoc Networks with Beamforming Antennas, In the Proceedings of ACM Symposium on Mobile Ad hoc Networking and Computing (MobiHoc'2001), (2001).

[2] S. Yi, Y. Pei and S. Kalyanaraman, On the capacity improvement of ad hoc wireless networks using directional antennas. Proceedings of ACM MobiHoc, (2003).

[3] J. Zhang, X. Jia, Capacity analysis of wireless mesh networks with omni or directional antennas. IEEE INFOCOM (mini-conference), (2009)

[4] M. Takata, K. Nagashima and T. Watanabe, A dual access mode MAC protocol for ad hoc networks using smart antennas. Proceedings of IEEE ICC, (2004).

[5] T. Ohira, K. Iigusa, Electronically Steerable Parastic Array Radiator Antenna, IEICE Trans. Commun., January (2004), Vol.J87-C, No.1, pp12-31, Japan.

[6] H. N. Dai, K. W. Ng, R. C. W. Wong and M.Y. Wu, On the capacity of multi-channel wireless networks using directional antennas. Proceedings of IEEE INFOCOM, (2008).

[7] S. S. Kushwah, S. S. Bhadoria and G .S. Tomar, Analysis of DMAC Protocols in Wireless Ad Hoc Networks, International Journal of Communication Systems and Networks, (2012), Vol.1 No.1, pp.102-109.

[8] S. Kushwah, G. S. Tomar and S. S. Bhadoria, Analysis of DMAC Protocols in Wireless Ad hoc Networks, International Journal of Communication Systems and Network Technologies, (2012), Vol.1, No.2. pp.97-103.

[9] A. S. Tomar and G. S. Tomar, Improved DMAC for Multi Hop Wireless Ad hoc Networks, IEEE International Conference CICN 2013, 27-29 Sep. (2013), pp 97-99.

[10] G. S. Tomar, S. Verma and M. Rai; Modified DMAC for ad hoc Networks using Bootlace Lens, IEEE International Conference, TENCON-2006, 14-17 Nov (2006), pp-4.

[11] S. Suhag, A. Gupta and M. Duhan, Improvement of QoS Parameters by using Directional Antennas in MANET, International Journal of Advanced Research in Computer Science and Software Engineering, (2015), Vol.5, No.6, pp.1025-1031.

[12] H. N. Dai, K. W. Ng, M. Li and M. Y. Wu, An Overview of Using Directional Antennas in Wireless Networks, Int. J. Commun. Syst. (2011).

[13] L. Shrivastava, S. S. Bhadauria and G. S. Tomar, Influence of Traffic Load on the performance of AODV, DSR and DSDV in MANET, International Journal of Communication Systems and Network Technologies, 
(2013), Vol.1 Issue 1. pp 22-34.

[14] G. S Tomar, T. Sharma, D. Bhattacharyya and T. H. Kim, Proceedings of the 2011 International Conference on Ubiquitous Computing and Multimedia Applications, (2011), pp 3-7. 
Performance Analysis of Wireless Mobile Adhoc Network with Different Types of Antennas

(This page is empty intentionally) 